\title{
Compact Dynamic Modeling for Fast Simulation of Nonlinear Heat Conduction in Ultra-Thin Chip Stacking Technology
}

\author{
Lorenzo Codecasa, Member, IEEE, Vincenzo d'Alessandro, Alessandro Magnani, \\ and Niccolò Rinaldi, Member, IEEE
}

\begin{abstract}
A novel nonlinear model order reduction method is proposed for constructing one-port dynamic compact models of nonlinear heat diffusion problems for ultra-thin chip stacking technology. The method leads to models of small state-space dimensions, which allow accurately reconstructing the whole time evolution of the temperature field due to an arbitrary power waveform of practical interest. The approach is also efficient, since the computational time/memory requirements for constructing each dynamic compact model is about one order of magnitude lower than that corresponding to a single 3-D finite element method transient simulation of a nonlinear problem.
\end{abstract}

Index Terms-Dynamic compact thermal model (DCTM), nonlinear model order reduction (MOR), self-heating, thermal impedance, ultra-thin chip stacking (UTCS).

\section{INTRODUCTION}

$\mathbf{T}$ HERMAL management is becoming crucial for stateof-the-art highly-integrated electronic systems, which are adversely impacted by over-heating in both performance and reliability. Unfortunately, the design of such systems is typically supported by computationally onerous numerical simulations relying on the finite difference or finite element methods (FEM), since analytical solutions of the heat equation are available only for over-simplified cases. Consequently, in the last decades, a considerable effort has been made to develop dynamic compact thermal models (DCTMs) which, once constructed, allow determining the time evolution of the temperatures of the electronic components at a much lower cost than conventional numerical approaches [1]- [4].

Particularly attractive is the Model Order Reduction (MOR) approach introduced by one of the authors in his MultiPoint Moment Matching (MPMM) technique [5]-[9]. Such method not only leads to accurate DCTMs of small statespace dimensions for estimating the time evolution of the temperatures of the electronic components, but, unlike other

Manuscript received February 11, 2014; revised July 2, 2014; accepted August 18, 2014. Date of publication September 22, 2014; date of current version October 31, 2014. Recommended for publication by Associate Editor N. Sabry upon evaluation of reviewers' comments.

L. Codecasa is with the Dipartimento di Elettronica, Informazione e Bioingegneria, Politecnico di Milano, Milan 20133, Italy (e-mail: lorenzo.codecasa@polimi.it).

V. d'Alessandro, A. Magnani, and N. Rinaldi are with the Department of Electrical Engineering and Information Technology, Univeristy Federico II, 80125 Naples, Italy (e-mail: vindales@unina.it; alessandro.magnani@unina.it; nirinald@unina.it). approaches, it also allows reconstructing the whole space-time evolution of the temperature field. Moreover, differently from other techniques, the construction of the DCTMs avoids long transient simulations, since it involves only a limited number of much faster frequency domain simulations of discretized linear heat diffusion equations.

Almost all the proposed approaches for constructing DCTMs apply only to linear thermal problems, in which the thermal properties of materials are assumed to be temperatureindependent. However, for many heat diffusion problems in electronic components, the temperature dependence of thermal conductivities cannot be neglected [10]. A relevant case, here considered, is that of electronic modules fabricated in ultra-thin chip stacking (UTCS) technology [11]-[13]. The commonly adopted approach for constructing DCTMs devised for nonlinear thermal problems is based on the Kirchhoff's transformation [14], [15], by which the solution to the nonlinear heat diffusion problem is mapped onto the solution to a linear problem. However, this approach in practice provides exact results only under steady-state conditions and assuming a single material for the whole geometrical domain, while introducing large inaccuracies in most cases, like in UTCS architectures.

In this paper, a novel MOR method inspired by previous approaches [16]-[19] is proposed for constructing DCTMs of nonlinear heat diffusion problems in UTCS technology. It stems from a novel reformulation of the nonlinear heat diffusion equations in a form the projection of which directly leads to a nonlinear compact model. Such projection is performed by preserving the nonlinear structure of the reformulated heat diffusion equations [20], [21]. The projection space is determined from a few moments of the Volterra's [16]-[22] series expansion of the solution to the nonlinear heat diffusion problem. Although this approach can in principle be used to solve the analytical nonlinear heat transfer equation, in this paper it is actually applied to the FEM discretization of this equation, which is crucial in industrial applications.

The method extends from the linear to the nonlinear case most of the advantages of the MPMM approach in terms of efficiency and accuracy, i.e., the extracted DCTMs are of small state-space dimensions and, once constructed, allow dramatically reducing the time/storage requirements in comparison with classic numerical approaches. The construction of the 
DCTMs is extremely fast since it requires the solution to a limited number of discretized linear equations in the complex frequency domain. The DCTMs are suited to determine the whole space-time evolution of the temperature field for arbitrarily complex domains and power profiles.

As a first investigation, the analysis focuses on the case of one-port DCTMs, variously analyzed in literature in the linear case [23] because of its practical relevance, corresponding to UTCS in which one independent power source is active. Such one-port DCTM includes the chosen boundary conditions, assumed of homogeneous Robin's type, and considers initial ambient temperature. The extension of the proposed approach for constructing multi-port DCTMs suited to describe UTCS in which multiple independent power sources are concurrently active, as well as DCTMs independent of boundary and initial conditions [2]-[4], is currently under investigation.

This paper is organized as follows. In Section II, after discussing the limitations of the Kirchhoff's transformation, the nonlinear heat diffusion problem is reformulated in a novel way. In Section III, it is shown how DCTMs can be derived by projecting the reformulated heat diffusion equations in a way that preserves their nonlinear structure. In Section IV, the projection space is determined by computing the first moments of the Volterra's series expansions for the solution to the nonlinear heat diffusion problem, leading to a DCTM suited to evaluate the temperature rise in the whole physical domain for an arbitrarily shaped power profile. In Section V, the proposed procedure is applied to perform an extensive analysis of the nonlinear heat diffusion problem in a highlyintegrated electronic UTCS system.

\section{REFORMULATION OF THE NONLINEAR HeAT DifFusion PROBlem}

A nonlinear heat diffusion problem, in the spatial domain $\Omega$, is ruled by

$$
\begin{aligned}
& \rho(\mathbf{r}, \vartheta(\mathbf{r}, t)) c(\mathbf{r}, \vartheta(\mathbf{r}, t)) \frac{\partial \vartheta}{\partial t}(\mathbf{r}, t)+ \\
& \quad+\nabla \cdot[-k(\mathbf{r}, \vartheta(\mathbf{r}, t)) \nabla \vartheta(\mathbf{r}, t)]=q(\mathbf{r}, t)
\end{aligned}
$$

in which the unknown $\vartheta(\mathbf{r}, t)$, function of the position vector $\mathbf{r}$ and of the time instant $t$, is the temperature rise with respect to ambient temperature $T_{0}=300 \mathrm{~K}$ due to the power density $q(\mathbf{r}, t)$. In general, the mass density $\rho(\mathbf{r}, \vartheta(\mathbf{r}, t))$, the specific heat $c(\mathbf{r}, \vartheta(\mathbf{r}, t))$, and the thermal conductivity $k(\mathbf{r}, \vartheta(\mathbf{r}, t))$ depend on the temperature rise $\vartheta(\mathbf{r}, t)$. Robin conditions are assumed on the boundary $\partial \Omega$ of $\Omega$, in the form

$$
-k(\mathbf{r}, \vartheta(\mathbf{r}, t)) \frac{\partial \vartheta}{\partial n}(\mathbf{r}, t)=h(\mathbf{r}, \vartheta(\mathbf{r}, t)) \vartheta(\mathbf{r}, t)
$$

in which $\mathbf{n}(\mathbf{r})$ is the outward normal unit vector, and $h(\mathbf{r}, \vartheta(\mathbf{r}, t))$ is the heat transfer coefficient, which in general is temperature-dependent. Due to the difficulty in estimating the heat transfer coefficients, often either Neumann or Dirichlet homogeneous boundary conditions are preferred, which can be obtained from (2) by taking the limits $h(\mathbf{r}) \rightarrow 0$ and $h(\mathbf{r}) \rightarrow \infty$, respectively. Homogeneous initial conditions are considered

$$
\vartheta(\mathbf{r}, 0)=0 \text {. }
$$

A thermal model can be defined as usual [9]. Thus, for the one-port case considered hereinafter, the power density $q(\mathbf{r}, t)$ is written in the form

$$
q(\mathbf{r}, t)=g(\mathbf{r}) P(t)
$$

in which $P(t)$ is the power dissipated by the active heat source and injected at the port of the thermal model, while

$$
T(t)=T_{0}+\int_{\Omega} g(\mathbf{r}) \vartheta(\mathbf{r}, t) d \mathbf{r}
$$

defines the average temperature of the active heat source, measured at the port of the thermal model. It is noted that the normalized thermal response to a dissipated power step $P$ is introduced as

$$
Z_{\mathrm{TH}}(t)=\frac{T(t)-T_{0}}{P} .
$$

Following a common practice, $Z_{\mathrm{TH}}(t)$ is referred to as thermal impedance and its steady-state value is denoted as thermal resistance and indicated by $R_{\mathrm{TH}}$.

The most common approach for determining a DCTM for a nonlinear heat diffusion problem exploits the Kirchhoff's transformation [24]. In such an approach, the thermal parameters are assumed to take the form

$$
\begin{aligned}
& \rho(\mathbf{r}, \vartheta(\mathbf{r}, t)) c(\mathbf{r}, \vartheta(\mathbf{r}, t))=\rho(\mathbf{r}, 0) c(\mathbf{r}, 0) f(\vartheta(\mathbf{r}, t)) \\
& k(\mathbf{r}, \vartheta(\mathbf{r}, t))=k(\mathbf{r}, 0) f(\vartheta(\mathbf{r}, t)) \\
& h(\mathbf{r}, \vartheta(\mathbf{r}, t))=h(\mathbf{r}, 0) \frac{\int_{0}^{\vartheta(\mathbf{r}, t)} f\left(\vartheta^{\prime}\right) d \vartheta^{\prime}}{\vartheta(\mathbf{r}, t)}
\end{aligned}
$$

being $f(\cdot)$ a given positive function. In this case, by the change of variable

$$
u(\mathbf{r}, t)=\int_{0}^{\vartheta(\mathbf{r}, t)} f\left(\vartheta^{\prime}\right) d \vartheta^{\prime}=F(\vartheta(\mathbf{r}, t))
$$

the nonlinear heat diffusion problem is mapped onto the linear heat diffusion equation

$$
\rho(\mathbf{r}, 0) c(\mathbf{r}, 0) \frac{\partial u}{\partial t}(\mathbf{r}, t)+\nabla \cdot[-k(\mathbf{r}, 0) \nabla u(\mathbf{r}, t)]=q(\mathbf{r}, t)
$$

with linear boundary conditions

$$
-k(\mathbf{r}, 0) \frac{\partial u}{\partial n}(\mathbf{r}, t)=h(\mathbf{r}, 0) u(\mathbf{r}, t)
$$

and linear initial conditions

$$
u(\mathbf{r}, 0)=0 .
$$

From the solution to this linear heat diffusion problem, the solution to the nonlinear problem is simply achieved by inverting the Kirchhoff's transformation (7). As a result, a DCTM of the nonlinear heat diffusion problem (1), (2) is obtained by determining a DCTM of the linear heat diffusion problem (8), (9) by any suitable technique such as the MPMM approach [6], and inverting (7).

However, when considering electronic modules fabricated in UTCS technology, the thermal conductivity of the materials is commonly assumed to depend on $\vartheta(\mathbf{r}, t)$ according to

$$
k(\mathbf{r}, \vartheta(\mathbf{r}, t))=k_{1}(\mathbf{r})\left(1+\frac{\vartheta(\mathbf{r}, t)}{T_{0}}\right)^{m(\mathbf{r})}+k_{2}(\mathbf{r})
$$


in which parameters $k_{1}(\mathbf{r}), k_{2}(\mathbf{r})$, and $m(\mathbf{r})$ have different values in the individual layers. Besides, the specific heat $c(\mathbf{r})$, the mass density $\rho(\mathbf{r})$, and the heat transfer coefficient $h(\mathbf{r})$ are commonly assumed to be temperature-independent. In such a case, the Kirchhoff's transformation approach does not rigorously apply.

A practical widely-adopted solution is to assume a spatially uniform temperature dependence for all material thermal parameters, of the form (11) with $k_{2}=0$ and $m$ considered independent of the position vector $\mathbf{r}$, and to apply the Kirchhoff's transformation approach. In this way, $f\left(\vartheta^{\prime}\right)=\left(1+\vartheta^{\prime} / T_{0}\right)^{m}$ so that Kirchhoff's transformation is

$$
u(\mathbf{r}, t)=F(\vartheta(\mathbf{r}, t))=\frac{T_{0}}{m+1}\left[\left(1+\frac{\vartheta(\mathbf{r}, t)}{T_{0}}\right)^{m+1}-1\right] .
$$

Its inverse

$$
\vartheta(\mathbf{r}, t)=T_{0}\left[\left(1+\frac{m+1}{T_{0}} u(\mathbf{r}, t)\right)^{\frac{1}{m+1}}-1\right]
$$

can thus be used for transforming the solution to the linear heat diffusion equations (8)-(10) into an approximation of the solutions to the nonlinear heat diffusion equations (1)-(3). As an extension to this approach, the transformation proposed by Batty et al. in [25]-[27] can be also used. In this method, first, the transformed temperature rise $v(\mathbf{r}, \tau)$ is determined as the solution to the linear heat diffusion equations obtained from (8)-(10) by substituting $t$ with $\tau$ and $u(\mathbf{r}, t)$ with $v(\mathbf{r}, \tau)$. Second, the time variable $t$ is reconstructed from $\tau$ by

$$
t=\int_{0}^{\tau}\left(1+\frac{m+1}{T_{0}} v\left(\mathbf{r}, \tau^{\prime}\right)\right)^{-\frac{m}{m+1}} d \tau^{\prime}
$$

and the transformed temperature rise $u(\mathbf{r}, t)=v(\mathbf{r}, \tau)$ is computed. Last, the inverse Kirchhoff's transformation (12) is used to map $u(\mathbf{r}, t)$ into an approximation of the temperature rise $\vartheta(\mathbf{r}, t)$.

As numerically shown in Section V, in UTCS modules both these transformations introduce large inaccuracies that cannot be controlled. For this reason, a rigorous, alternative strategy to dynamic compact thermal modeling of nonlinear heat diffusion is highly desired.

In this paper, we propose a novel approach, which is not based on the aforementioned transformations but in which the nonlinear heat diffusion problem is reformulated in an equivalent way. In particular, by introducing the additional variable

$$
\lambda(\mathbf{r}, t)=\left(1+\frac{\vartheta(\mathbf{r}, t)}{T_{0}}\right)^{m(\mathbf{r})}-1,
$$

equations (1) and (2) are rewritten in the form

$$
\begin{gathered}
\rho(\mathbf{r}) c(\mathbf{r}) \frac{\partial \vartheta}{\partial t}(\mathbf{r}, t)+\nabla \cdot\left[-\left(k_{0}(\mathbf{r})+k_{1}(\mathbf{r}) \lambda(\mathbf{r}, t)\right) \nabla \vartheta(\mathbf{r}, t)\right] \\
=g(\mathbf{r}) P(t) \\
-\left(k_{0}(\mathbf{r})+k_{1}(\mathbf{r}) \lambda(\mathbf{r}, t)\right) \frac{\partial \vartheta}{\partial n}(\mathbf{r}, t)=h(\mathbf{r}) \vartheta(\mathbf{r}, t)
\end{gathered}
$$

being $k_{0}(\mathbf{r})=k_{1}(\mathbf{r})+k_{2}(\mathbf{r})$. Besides, by deriving (13) with respect to time, it is obtained

$$
\left(T_{0}+\vartheta(\mathbf{r}, t)\right) \frac{\partial \lambda}{\partial t}(\mathbf{r}, t)=m(\mathbf{r})(1+\lambda(\mathbf{r}, t)) \frac{\partial \vartheta}{\partial t}(\mathbf{r}, t) .
$$

Equation (16) is equivalent to (13), when the initial condition

$$
\lambda(\mathbf{r}, 0)=0
$$

is introduced. As a result, the nonlinear heat diffusion problem (1)-(3) is reformulated by (3) and (14)-(17) in the following. The thermal model is still defined by (4) and (5).

\section{Structure-Preserving Compact Modeling}

A DCTM is here achieved from the nonlinear heat diffusion equations, as reformulated in Section II, by a novel projection approach which preserves their nonlinear structure. To this aim, $\vartheta(\mathbf{r}, t)$ is approximated in the form

$$
\vartheta(\mathbf{r}, t)=\sum_{j=1}^{\hat{n}_{\vartheta}} \vartheta_{j}(\mathbf{r}) \hat{\vartheta}_{j}(t)
$$

in which $\vartheta_{j}(\mathbf{r})$, with $j=1, \ldots, \hat{n}_{\vartheta}$, are a small number of basis functions, which will be determined in Section IV. Likewise, $\lambda(\mathbf{r}, t)$ is approximated in the form

$$
\lambda(\mathbf{r}, t)=\sum_{k=1}^{\hat{n}_{\lambda}} \lambda_{k}(\mathbf{r}) \hat{\lambda}_{k}(t)
$$

in which $\lambda_{k}(\mathbf{r})$, with $k=1, \ldots, \hat{n}_{\lambda}$, are a small number of basis functions, which again will be determined in Section IV. It is noted that the number $\hat{n}_{\vartheta}$ of the basis functions $\vartheta_{j}(\mathbf{r})$ and the number $\hat{n}_{\lambda}$ of the basis functions $\lambda_{j}(\mathbf{r})$ are not necessarily equal.

By multiplying (14) by $\vartheta_{i}(\mathbf{r})$, integrating over $\Omega$, applying the divergence theorem and recalling (15), (18), and (19), the following equation is obtained:

$$
\begin{array}{r}
\sum_{j=1}^{\hat{n}_{\vartheta}} \hat{c}_{i j}^{0} \frac{d \hat{\vartheta}_{j}}{d t}(t)+\sum_{j=1}^{\hat{n}_{\vartheta}} \hat{k}_{i j}^{0} \hat{\vartheta}_{j}(t)+\sum_{j=1}^{\hat{n}_{\vartheta}} \sum_{k=1}^{\hat{n}_{\lambda}} \hat{k}_{i j k}^{1} \hat{\vartheta}_{j}(t) \hat{\lambda}_{k}(t) \\
=\hat{g}_{i} P(t)
\end{array}
$$

in which

$$
\begin{aligned}
& \hat{c}_{i j}^{0}=\int_{\Omega} \rho(\mathbf{r}) c(\mathbf{r}) \vartheta_{i}(\mathbf{r}) \vartheta_{j}(\mathbf{r}) d \mathbf{r} \\
& \hat{k}_{i j}^{0}=\int_{\Omega} k_{0}(\mathbf{r}) \nabla \vartheta_{i}(\mathbf{r}) \cdot \nabla \vartheta_{j}(\mathbf{r}) d \mathbf{r}+\int_{\partial \Omega} h(\mathbf{r}) \vartheta_{i}(\mathbf{r}) \vartheta_{j}(\mathbf{r}) d \mathbf{r} \\
& \hat{k}_{i j k}^{1}=\int_{\Omega} k_{1}(\mathbf{r}) \lambda_{k}(\mathbf{r}) \nabla \vartheta_{i}(\mathbf{r}) \cdot \nabla \vartheta_{j}(\mathbf{r}) d \mathbf{r} \\
& \hat{g}_{i}=\int_{\Omega} g(\mathbf{r}) \vartheta_{i}(\mathbf{r}) d \mathbf{r} .
\end{aligned}
$$

Similarly, multiplying (16) by $\lambda_{i}(\mathbf{r})$, integrating over $\Omega$ and recalling (18) and (19), it is found

$$
\begin{aligned}
T_{0} \sum_{k=1}^{\hat{n}_{\lambda}} \hat{\gamma}_{i k}^{0} \frac{d \hat{\lambda}_{k}}{d t}(t)+\sum_{j=1}^{\hat{n}_{\vartheta}} \sum_{k=1}^{\hat{n}_{\lambda}} \hat{\gamma}_{i j k}^{1} \hat{\vartheta}_{j}(t) \frac{d \hat{\lambda}_{k}}{d t}(t) \\
=\sum_{j=1}^{\hat{n}_{\vartheta}} \hat{\mu}_{i j}^{0} \frac{d \hat{\vartheta}_{j}}{d t}(t)+\sum_{j=1}^{\hat{n}_{\vartheta}} \sum_{k=1}^{\hat{n}_{\lambda}} \hat{\mu}_{i j k}^{1} \frac{d \hat{\vartheta}_{j}}{d t}(t) \hat{\lambda}_{k}(t)
\end{aligned}
$$


in which

$$
\begin{aligned}
\hat{\gamma}_{i k}^{0} & =\int_{\Omega} \lambda_{i}(\mathbf{r}) \lambda_{k}(\mathbf{r}) d \mathbf{r} \\
\hat{\gamma}_{i j k}^{1} & =\int_{\Omega} \lambda_{i}(\mathbf{r}) \lambda_{k}(\mathbf{r}) \vartheta_{j}(\mathbf{r}) d \mathbf{r} \\
\hat{\mu}_{i j}^{0} & =\int_{\Omega} m(\mathbf{r}) \lambda_{i}(\mathbf{r}) \vartheta_{j}(\mathbf{r}) d \mathbf{r} \\
\hat{\mu}_{i j k}^{1} & =\int_{\Omega} m(\mathbf{r}) \lambda_{i}(\mathbf{r}) \lambda_{k}(\mathbf{r}) \vartheta_{j}(\mathbf{r}) d \mathbf{r} .
\end{aligned}
$$

Also, from (3) and (17), the initial conditions for the compact model follow

$$
\begin{aligned}
& \hat{\vartheta}_{i}(t)=0, \quad i=1, \ldots, \hat{n}_{\vartheta} \\
& \hat{\lambda}_{k}(t)=0, \quad k=1, \ldots, \hat{m}_{\lambda} .
\end{aligned}
$$

From (5) and (18), the DCTM defined by (20) and (23) leads to an approximation of the temperature $T(t)$ of the active heat source given by

$$
\hat{T}(t)=T_{0}+\sum_{j=1}^{\hat{n}_{\vartheta}} \hat{g}_{j} \hat{\vartheta}_{j}(t)
$$

in which $\hat{g}_{i}$ are defined by (22). As a post-processing, it allows reconstructing the whole temperature rise evolution $\vartheta(\mathbf{r}, t)$ within $\Omega$ in the form

$$
\hat{\vartheta}(\mathbf{r}, t)=\sum_{j=1}^{\hat{n}_{\vartheta}} \vartheta_{j}(\mathbf{r}) \hat{\vartheta}_{j}(t) .
$$

It is noted that the novel projection method here adopted preserves the nonlinear structure of the heat diffusion problem, as reformulated in Section II. As a result, the nonlinear DCTM is expected to match the qualitative properties of the nonlinear heat diffusion problem. A quantitative match can be achieved by choosing the basis functions $\vartheta_{j}(\mathbf{r})$, with $j=1, \ldots, \hat{n}_{\vartheta}$ and $\lambda_{j}(\mathbf{r})$, with $j=1, \ldots, \hat{n}_{\lambda}$ as described in Section IV.

\section{BASIS FUNCTIONS FROM VOLTERRA'S SERIES MOMENTS}

As it is well known, for a single-input single-output timeinvariant nonlinear dynamic system, under proper regularity conditions, the input $u(t)$ and output $y(t)$ are related by a Volterra's series expansion in the form

$$
\begin{aligned}
y(t)= & \sum_{m=1}^{\infty} \int_{0}^{+\infty} \cdots \int_{0}^{+\infty} h_{m}\left(t_{1}, t_{2}, \ldots, t_{m}\right) u\left(t-t_{1}\right) u\left(t-t_{2}\right) \\
& \times \cdots u\left(t-t_{m}\right) d t_{1} d t_{2} \ldots d t_{m}
\end{aligned}
$$

in which $h_{m}\left(t_{1}, t_{2}, \ldots, t_{m}\right)$ are the $m$-th-order Volterra kernels, assumed to be symmetric with respect to $t_{1}, t_{2}, \ldots, t_{m}$. By taking the multidimensional Laplace transforms of $h_{m}\left(t_{1}, t_{2}, \ldots, t_{m}\right)$, it is obtained

$$
\begin{aligned}
& H_{m}\left(s_{1}, s_{2}, \ldots, s_{m}\right)=\int_{0}^{+\infty} \ldots \\
& \quad \times \int_{0}^{+\infty} h_{m}\left(t_{1}, t_{2}, \ldots, t_{m}\right) e^{-\left(s_{1} t_{1}+s_{2} t_{2}+\cdots+s_{m} t_{m}\right)} d t_{1} d t_{2} \ldots d t_{m} .
\end{aligned}
$$

The Laplace transforms $U(s), Y(s)$ of $u(t), y(t)$, respectively, are then related by

$$
\begin{aligned}
Y(s) & =\sum_{m=1}^{\infty} \frac{1}{(2 \pi i)^{m-1}} \int_{\sigma_{1}-i \infty}^{\sigma_{1}+i \infty} \ldots \\
& \times \int_{\sigma_{m}-i \infty}^{\sigma_{m}+i \infty} Y_{m}\left(s-s_{1} \ldots-s_{m-1}, s_{1}, \ldots, s_{m-1}\right) d s_{1} \ldots d s_{m-1}
\end{aligned}
$$

in which

$Y_{m}\left(s_{1}, s_{2}, \ldots, s_{m}\right)=H_{m}\left(s_{1}, s_{2}, \ldots, s_{m}\right) U\left(s_{1}\right) U\left(s_{2}\right) \cdots U\left(s_{m}\right)$.

As a result, the output variable $y(t)$ can be recovered from the Volterra's series terms $Y_{m}\left(s_{1}, s_{2}, \ldots, s_{m}\right)$, which can be assumed to be symmetric with respect to $s_{1}, s_{2}, \ldots, s_{m}$.

Such Volterra's series terms are now considered for the solution to the nonlinear heat diffusion problem. To this aim, by introducing the additional variables $\varphi(\mathbf{r}, t), \omega(\mathbf{r}, t)$

$$
\begin{aligned}
& \frac{\partial \vartheta}{\partial t}(\mathbf{r}, t)=\varphi(\mathbf{r}, t) \\
& \frac{\partial \lambda}{\partial t}(\mathbf{r}, t)=\omega(\mathbf{r}, t)
\end{aligned}
$$

(14)-(16) are rewritten, respectively, in the form

$$
\begin{gathered}
\rho(\mathbf{r}) c(\mathbf{r}) \varphi(\mathbf{r}, t)+\nabla \cdot\left[-\left(k_{0}(\mathbf{r})+k_{1}(\mathbf{r}) \lambda(\mathbf{r}, t)\right) \nabla \vartheta(\mathbf{r}, t)\right] \\
=g(\mathbf{r}) P(t) \\
-\left(k_{0}(\mathbf{r})+k_{1}(\mathbf{r}) \lambda(\mathbf{r}, t)\right) \frac{\partial \vartheta}{\partial n}(\mathbf{r}, t)=h(\mathbf{r}) \vartheta(\mathbf{r}, t) \\
\left(T_{0}+\vartheta(\mathbf{r}, t)\right) \omega(\mathbf{r}, t)=m(\mathbf{r})(1+\lambda(\mathbf{r}, t)) \varphi(\mathbf{r}, t)
\end{gathered}
$$

Then, Volterra's series expansion terms $\Theta_{m}\left(\mathbf{r}, \mathbf{s}_{m}\right), \Phi_{m}\left(\mathbf{r}, \mathbf{s}_{m}\right)$, $\Lambda_{m}\left(\mathbf{r}, \mathbf{s}_{m}\right), \Omega_{m}\left(\mathbf{r}, \mathbf{s}_{m}\right)$ are computed for $\vartheta(\mathbf{r}, t), \varphi(\mathbf{r}, t)$, $\lambda(\mathbf{r}, t), \omega(\mathbf{r}, t)$, respectively, in which the notation $\mathbf{s}_{m}=$ $\left(s_{1}, s_{2}, \ldots, s_{m}\right)$ is adopted. Using well-known properties of the theory of Volterra's series expansion [22], [28], from (26)-(29) it follows:

$$
\begin{aligned}
& \left|\mathbf{s}_{m}\right| \Theta_{m}\left(\mathbf{r}, \mathbf{s}_{m}\right)=\Phi_{m}\left(\mathbf{r}, \mathbf{s}_{m}\right) \\
& \left|\mathbf{s}_{m}\right| \Lambda_{m}\left(\mathbf{r}, \mathbf{s}_{m}\right)=\Omega_{m}\left(\mathbf{r}, \mathbf{s}_{m}\right) .
\end{aligned}
$$

and (33)-(35), as shown at the top of the next page.

In these expressions, $\boldsymbol{p}_{m}$ is a vector of $m$ elements equal to either zero or one, $\left|\boldsymbol{p}_{m}\right|$ indicates the sum of the elements of vector $\boldsymbol{p}_{m}$, and $\boldsymbol{p}_{m}^{\prime}$ is the vector obtained from $\boldsymbol{p}_{m}$ by exchanging all zeros with ones. Also, $\mathbf{s}_{m}^{\boldsymbol{p}_{m}}$ is the vector obtained by selecting the elements of $\mathbf{s}_{m}$ corresponding to the ones of $\boldsymbol{p}_{m}$. The iterative solution of these equations, for $m=1,2, \ldots$ determine all Volterra's series expansion terms $\Theta_{m}\left(\mathbf{r}, \mathbf{s}_{m}\right), \Phi_{m}\left(\mathbf{r}, \mathbf{s}_{m}\right), \Lambda_{m}\left(\mathbf{r}, \mathbf{s}_{m}\right), \Omega_{m}\left(\mathbf{r}, \mathbf{s}_{m}\right)$.

Multidimensional Taylor series expansions of $\Theta_{m}\left(\mathbf{r}, \mathbf{s}_{m}\right)$ can be written around any chosen value $\sigma_{m}$ of $\mathbf{s}_{m}$ in the form

$$
\Theta_{m}\left(\mathbf{r}, \mathbf{s}_{m}\right)=\sum_{\boldsymbol{\alpha}_{m}} \Theta_{m, \boldsymbol{\alpha}_{m}}\left(\mathbf{r}, \boldsymbol{\sigma}_{m}\right)\left(\mathbf{s}_{m}-\boldsymbol{\sigma}_{m}\right)^{\boldsymbol{\alpha}_{m}}
$$

in which $\boldsymbol{\alpha}_{m}$ are all multi-indexes of $m$ elements and $\Theta_{m, \boldsymbol{\alpha}_{m}}\left(\mathbf{r}, \boldsymbol{\sigma}_{m}\right)$ are the moments of $\Theta_{m}\left(\mathbf{r}, \mathbf{s}_{m}\right)$. Similar series expansions around $\sigma_{m}$ can be introduced for the other quantities $\Phi_{m}\left(\mathbf{r}, \mathbf{s}_{m}\right), \Lambda_{m}\left(\mathbf{r}, \mathbf{s}_{m}\right)$ and $\Omega_{m}\left(\mathbf{r}, \mathbf{s}_{m}\right)$.

In order to determine the basis functions $\vartheta_{j}(\mathbf{r})$, with $j=1, \ldots, \hat{n}_{\vartheta}$, and $\lambda_{j}(\mathbf{r})$, with $j=1, \ldots, \hat{n}_{\lambda}$, a set 


$$
\begin{aligned}
& \rho(\mathbf{r}) c(\mathbf{r}) \Phi_{m}\left(\mathbf{r}, \mathbf{s}_{m}\right)+\nabla \cdot\left[-k_{0}(\mathbf{r}) \nabla \Theta_{m}\left(\mathbf{r}, \mathbf{s}_{m}\right)\right]+\sum_{k=1}^{m-1} \nabla \cdot\left[-k_{1}(\mathbf{r}) \sum_{\mid \boldsymbol{p}_{m} \models k} \Lambda_{k}\left(\mathbf{r}, \mathbf{s}_{m}^{\boldsymbol{p}_{m}}\right) \nabla \Theta_{m-k}\left(\mathbf{r}, \mathbf{s}_{m}^{\boldsymbol{p}_{m}^{\prime}}\right)\right] /\left(\begin{array}{l}
m \\
k
\end{array}\right)=g(\mathbf{r}) \delta_{m 1} \\
& -k_{0}(\mathbf{r}) \frac{\partial \Theta_{m}}{\partial n}\left(\mathbf{r}, \mathbf{s}_{m}\right)-\sum_{k=1}^{m-1} k_{1}(\mathbf{r}) \sum_{\left|\boldsymbol{p}_{m}\right|=k} \Lambda_{k}\left(\mathbf{r}, \mathbf{s}_{m}^{\boldsymbol{p}_{m}}\right) \frac{\partial \Theta_{m-k}}{\partial n}\left(\mathbf{r}, \mathbf{s}_{m}^{\boldsymbol{p}_{m}^{\prime}}\right) /\left(\begin{array}{l}
m \\
k
\end{array}\right)=h(\mathbf{r}) \Theta_{m}\left(\mathbf{r}, \mathbf{s}_{m}\right) \\
& \Omega_{m}\left(\mathbf{r}, \mathbf{s}_{m}\right)+\sum_{k=1}^{m-1} \sum_{\left|\boldsymbol{p}_{m}\right|=k} \Theta_{k}\left(\mathbf{r}, \mathbf{s}_{m}^{\boldsymbol{p}_{m}}\right) \Omega_{m-k}\left(\mathbf{r}, \mathbf{s}_{m}^{\prime}\right) /\left(\begin{array}{l}
m \\
k
\end{array}\right)= \\
& =m(\mathbf{r}) \Phi_{m}\left(\mathbf{r}, \mathbf{s}_{m}\right)+m(\mathbf{r}) \sum_{k=1}^{m-1} \sum_{\left|\boldsymbol{p}_{m}\right|=k} \Lambda_{k}\left(\mathbf{r}, \mathbf{s}_{m}^{\boldsymbol{p}_{m}}\right) \Phi_{m-k}\left(\mathbf{r}, \mathbf{s}_{m}^{\boldsymbol{p}_{m}^{\prime}}\right) /\left(\begin{array}{l}
m \\
k
\end{array}\right) . \\
& \left|\boldsymbol{\sigma}_{m}\right| \rho(\mathbf{r}) c(\mathbf{r}) \Theta_{m, \boldsymbol{\alpha}_{m}}\left(\mathbf{r}, \boldsymbol{\sigma}_{m}\right)+\nabla \cdot\left[-k_{0}(\mathbf{r}) \nabla \Theta_{m, \boldsymbol{\alpha}_{m}}\left(\mathbf{r}, \boldsymbol{\sigma}_{m}\right)\right]=-\sum_{k=1}^{m} \rho(\mathbf{r}) c(\mathbf{r}) \Theta_{m, \boldsymbol{\alpha}_{m}-\boldsymbol{e}_{m, k}}\left(\mathbf{r}, \boldsymbol{\sigma}_{m}\right)+ \\
& -\sum_{k=1}^{m-1} \nabla \cdot\left[-k_{1}(\mathbf{r}) \sum_{\left|\boldsymbol{p}_{m}\right|=k} \Lambda_{k, \boldsymbol{\alpha}_{m}^{\boldsymbol{p}_{m}}}\left(\mathbf{r}, \boldsymbol{\sigma}_{m}^{\boldsymbol{p}_{m}}\right) \nabla \Theta_{m-k, \boldsymbol{\alpha}_{m}^{\boldsymbol{p}_{m}^{\prime}}}\left(\mathbf{r}, \boldsymbol{\sigma}_{m}^{\boldsymbol{p}_{m}^{\prime}}\right)\right] /\left(\begin{array}{l}
m \\
k
\end{array}\right)+g(\mathbf{r}) \delta_{m 1} \delta_{\boldsymbol{\alpha}_{m} \mathbf{0}} \\
& -k_{0}(\mathbf{r}) \frac{\partial \Theta_{m, \boldsymbol{\alpha}_{m}}}{\partial n}\left(\mathbf{r}, \boldsymbol{\sigma}_{m}\right)-h(\mathbf{r}) \Theta_{m, \boldsymbol{\alpha}_{m}}\left(\mathbf{r}, \boldsymbol{\sigma}_{m}\right)=-\sum_{k=1}^{m-1} k_{1}(\mathbf{r}) \sum_{\left|\boldsymbol{p}_{m}\right|=k} \Lambda_{k, \boldsymbol{\alpha}_{m}^{\boldsymbol{p}_{m}}}\left(\mathbf{r}, \boldsymbol{\sigma}_{m}^{\boldsymbol{p}_{m}}\right) \frac{\partial \Theta_{m-k, \boldsymbol{\alpha}_{m}^{\boldsymbol{p}_{m}^{\prime}}}}{\partial n}\left(\mathbf{r}, \boldsymbol{\sigma}_{m}^{\boldsymbol{p}_{m}^{\prime}}\right) /\left(\begin{array}{c}
m \\
k
\end{array}\right) \\
& \Omega_{m, \boldsymbol{\alpha}_{m}}\left(\mathbf{r}, \boldsymbol{\sigma}_{m}\right)=-\sum_{k=1}^{m-1} \sum_{\left|\boldsymbol{p}_{m}\right|=k} \Theta_{k, \boldsymbol{\alpha}_{m}^{p_{m}}}\left(\mathbf{r}, \boldsymbol{\sigma}_{m}^{\boldsymbol{p}_{m}}\right) \Omega_{m-k, \boldsymbol{\alpha}_{m}^{\boldsymbol{p}_{m}^{\prime}}}\left(\mathbf{r}, \boldsymbol{\sigma}_{m}^{\boldsymbol{p}_{m}^{\prime}}\right) /\left(\begin{array}{l}
m \\
k
\end{array}\right)+ \\
& +m(\mathbf{r}) \Phi_{m, \boldsymbol{\alpha}_{m}}\left(\mathbf{r}, \boldsymbol{\sigma}_{m}\right)+m(\mathbf{r}) \sum_{k=1}^{m-1} \sum_{\left|\boldsymbol{p}_{m}\right|=k} \Lambda_{k, \boldsymbol{\alpha}_{m}^{\boldsymbol{p}_{m}}}\left(\mathbf{r}, \boldsymbol{\sigma}_{m}^{\boldsymbol{p}_{m}}\right) \Phi_{m-k, \boldsymbol{\alpha}_{m}^{\boldsymbol{p}_{m}^{\prime}}}\left(\mathbf{r}, \boldsymbol{\sigma}_{m}^{\boldsymbol{p}_{m}^{\prime}}\right) /\left(\begin{array}{l}
m \\
k
\end{array}\right)
\end{aligned}
$$

of expansion points $\sigma_{m}$ and expansion orders $q_{m}$ is now chosen with $m=1,2, \ldots, r$. By proceeding iteratively with $\left|\boldsymbol{\alpha}_{m}\right|=0,1, \ldots, q_{m}$ and $m=1,2, \ldots, r$, as a consequence of (31)-(35), moments $\Theta_{m, \boldsymbol{\alpha}_{m}}\left(\mathbf{r}, \boldsymbol{\sigma}_{m}\right)$ are determined by solving the linear heat diffusion problems in the frequency domain (36) and (37), as shown at the top of the page, in which the right hand sides (RHSs) are known from previous iterations. Moments $\Phi_{m, \boldsymbol{\alpha}_{m}}\left(\mathbf{r}, \boldsymbol{\sigma}_{m}\right)$ are explicitly determined as

$$
\begin{aligned}
\Phi_{m, \boldsymbol{\alpha}_{m}}\left(\mathbf{r}, \boldsymbol{\sigma}_{m}\right)= & \left|\boldsymbol{\sigma}_{m}\right| \Theta_{m, \boldsymbol{\alpha}_{m}}\left(\mathbf{r}, \boldsymbol{\sigma}_{m}\right)+ \\
& +\sum_{k=1}^{m} \Theta_{m, \boldsymbol{\alpha}_{m}-\boldsymbol{e}_{m, k}}\left(\mathbf{r}, \boldsymbol{\sigma}_{m}\right)
\end{aligned}
$$

in which the RHS is known. Moments $\Omega_{m, \boldsymbol{\alpha}_{m}}\left(\mathbf{r}, \boldsymbol{\sigma}_{m}\right)$ are evaluated as (39), as shown at the top of the page, in which the RHS is known. Lastly, moments $\Lambda_{m, \boldsymbol{\alpha}_{m}}\left(\mathbf{r}, \boldsymbol{\sigma}_{m}\right)$ are obtained from

$$
\begin{aligned}
& \Lambda_{m, \boldsymbol{\alpha}_{m}}\left(\mathbf{r}, \boldsymbol{\sigma}_{m}\right) \\
& =\left(\boldsymbol{\Omega}_{m, \boldsymbol{\alpha}_{m}}\left(\mathbf{r}, \boldsymbol{\sigma}_{m}\right)-\sum_{k=1}^{m} \Lambda_{m, \boldsymbol{\alpha}_{m}-\boldsymbol{e}_{m, k}}\left(\mathbf{r}, \boldsymbol{\sigma}_{m}\right)\right) /\left|\boldsymbol{\sigma}_{m}\right|
\end{aligned}
$$

in which again the RHS is known. The basis functions $\vartheta_{j}(\mathbf{r})$, with $j=1, \ldots, \hat{n}_{\vartheta}$ and $\lambda_{j}(\mathbf{r})$, with $j=1, \ldots, \hat{n}_{\lambda}$, are then determined as any linear combinations of the moments $\Theta_{m, \boldsymbol{\alpha}_{m}}\left(\mathbf{r}, \boldsymbol{\sigma}_{m}\right)$ and $\Lambda_{m, \boldsymbol{\alpha}_{m}}\left(\mathbf{r}, \boldsymbol{\sigma}_{m}\right)$, respectively, with $\left|\boldsymbol{\alpha}_{m}\right| \leq q_{m}$ and $m=1,2, \ldots, r$. It is noted that the chosen basis functions are not related to particular choices of the dissipated power $P(t)$, but to the local expansion of the solution to the nonlinear heat diffusion equation in the neighborhood of power $P(t)=0$. Despite the locality of this expansion, the resulting DCTMs turn out to be accurate up to values of the power $P(t)$ far above the largest significant levels, as verified by numerical experiments in Section V.

The following remarks can be made.

1) Only in exceptional situations, in which the geometries and material parameters are over-simplified, the linear heat diffusion equations (36) and (37) can be solved in closed form. This difficulty is overcome by numerically solving (36) and (37) through a discretization method. An efficient and accurate discretization method here used is FEM with second-order spatial approximation of the temperature rise $\Theta_{m, \boldsymbol{\alpha}_{m}}\left(\mathbf{r}, \boldsymbol{\sigma}_{m}\right)$ over a tetrahedral mesh. The resulting linear systems of equations, having symmetric, positive definite coefficient matrices, can be solved by various iterative solvers. The algorithm here used is the conjugate gradient algorithm with incomplete Choleski preconditioning. It is noted that the computational complexity of the entire approach is almost entirely due to the computational complexity of such iterative solver.

By introducing second-order spatial approximations also for the quantities $\Phi_{m, \boldsymbol{\alpha}_{m}}\left(\mathbf{r}, \boldsymbol{\sigma}_{m}\right), \boldsymbol{\Omega}_{m, \boldsymbol{\alpha}_{m}}\left(\mathbf{r}, \boldsymbol{\sigma}_{m}\right)$, and $\Lambda_{m, \boldsymbol{\alpha}_{m}}\left(\mathbf{r}, \boldsymbol{\sigma}_{m}\right)$, the solutions to (38)-(40) are simply achieved by evaluating (38)-(40) for the values of the position vector $\mathbf{r}$ at which the degrees of freedom 
TABLE I

VALUES OF THE EXPANSION POINTS $\boldsymbol{\sigma}_{m}$ AND MULTI-INDEXES $\boldsymbol{\alpha}_{m}$

\begin{tabular}{|l|l|l|}
\hline$m$ & $\boldsymbol{\sigma}_{m}$ & $\boldsymbol{\alpha}_{m}$ with $m=1, \ldots, r$ \\
\hline 1 & $\beta_{1}$ & 0 \\
& $\beta_{2}$ & 0 \\
& $\vdots$ & $\vdots$ \\
& $\beta_{l}$ & 0 \\
\hline 2 & $\left(\beta_{1}, \beta_{1}\right)$ & $(0,0)$ \\
& $\left(\beta_{2}, \beta_{1}\right)$ & $(0,0)$ \\
& $\vdots$ & $\vdots$ \\
& $\left(\beta_{l}, \beta_{1}\right)$ & $(0,0)$ \\
\hline$\vdots$ & $\vdots$ & $\vdots$ \\
\hline$r$ & $\left(\beta_{1}, \beta_{1}, \ldots, \beta_{1}\right)$ & $(0,0, \ldots, 0)$ \\
& $\left(\beta_{2}, \beta_{1}, \ldots, \beta_{1}\right)$ & $(0,0, \ldots, 0)$ \\
& $\vdots$ & $\vdots$ \\
& $\left(\beta_{l}, \beta_{1}, \ldots, \beta_{1}\right)$ & $(0,0, \ldots, 0)$ \\
\hline
\end{tabular}

(DoFs) of $\Theta_{m, \alpha_{m}}\left(\mathbf{r}, \boldsymbol{\sigma}_{m}\right), \Phi_{m, \boldsymbol{\alpha}_{m}}\left(\mathbf{r}, \boldsymbol{\sigma}_{m}\right), \Omega_{m, \boldsymbol{\alpha}_{m}}\left(\mathbf{r}, \boldsymbol{\sigma}_{m}\right)$, and $\Lambda_{m, \alpha_{m}}\left(\mathbf{r}, \boldsymbol{\sigma}_{m}\right)$ are set.

It is noted that the resulting equations for iteratively determining the discretizations of moments $\Theta_{m, \alpha_{m}}\left(\mathbf{r}, \boldsymbol{\sigma}_{m}\right), \quad \Phi_{m, \alpha_{m}}\left(\mathbf{r}, \boldsymbol{\sigma}_{m}\right), \quad \Omega_{m, \boldsymbol{\alpha}_{m}}\left(\mathbf{r}, \boldsymbol{\sigma}_{m}\right), \quad$ and $\Lambda_{m, \alpha_{m}}\left(\mathbf{r}, \sigma_{m}\right)$ are constructed at a much lower computational cost with respect to previous nonlinear MOR approaches using Volterra's series [16], [18], [19], in which computationally expensive Kronecker's tensor products have to be computed.

2) There is no strict prescription for choosing the expansion points $\sigma_{m}$ and matching orders $q_{m}$, with $m=1, \ldots, r$. An effective strategy is here adopted which extends the choice of expansion points used for linear heat diffusion problems in the MPMM approach. In MPMM, a set of $l$ real expansion points $\beta_{1}<\beta_{2}<\cdots \beta_{l}$ is introduced as detailed in [6]. Such expansion points are used for defining the set of expansion points $\sigma_{m}$ and multiindexes $\boldsymbol{\alpha}_{m}$, with $m=1, \ldots, r$ shown in Table I. As a result, in this way, $l r$ moments are determined by solving lr linear systems. As verified by numerical experiments in Section $\mathrm{V}$, in order to get accurate approximations of the space-time evolution of temperature, $l$ not larger than 15 and $r$ not larger than 4 can be assumed. As a result, the determination of a DCTM avoids long 3-D FEM transient simulations particularly demanding in the nonlinear case, while involving only a limited number of much faster simulations of 3-D FEM linear heat diffusion equations in the complex frequency domain.

3) In order to avoid ill conditioning of the DCTM, among all linear combinations of moments $\Theta_{m, \boldsymbol{\alpha}_{m}}\left(\mathbf{r}, \boldsymbol{\sigma}_{m}\right)$, with $\left|\boldsymbol{\alpha}_{m}\right| \leq q_{m}$ and $m=1, \ldots, r$, the basis functions $\vartheta_{j}(\mathbf{r})$, with $j=1, \ldots, \hat{n}_{\vartheta}$, are chosen as a set of orthonormal functions, so that

$$
\int_{\Omega} \vartheta_{i}(\mathbf{r}) \vartheta_{j}(\mathbf{r}) d \mathbf{r}=\delta_{i j}
$$

in which $\delta_{i j}$ is Kronecker's delta symbol. Such basis functions are here determined by applying the Singular Value Decomposition (SVD) algorithm as follows. Let $v_{i}(\mathbf{r})$, with $i=1, \ldots, \hat{n}$ be any sort of the chosen moments $\Theta_{m, \boldsymbol{\alpha}_{m}}\left(\mathbf{r}, \boldsymbol{\sigma}_{m}\right)$, with $\left|\boldsymbol{\alpha}_{m}\right| \leq q_{m}$ and $m=$ $1, \ldots, r$, and let $\mathbf{M}=\left[m_{i j}\right]$ be the matrix of order $\hat{n}$ given by

$$
m_{i j}=\int_{\Omega} v_{i}(\mathbf{r}) v_{j}(\mathbf{r}) d \mathbf{r} .
$$

Since $\mathbf{M}$ is real, symmetric, positive definite, it can be written in the form

$$
\mathbf{M}=\mathbf{U}^{T} \mathbf{D U}
$$

in which $\mathbf{U}=\left[u_{i j}\right]$ is a real orthogonal matrix of order $\hat{n}$ and $\mathbf{D}$ is a diagonal matrix with positive diagonal elements $d_{1} \leq d_{2} \leq \cdots d_{\hat{n}}$. The set of orthonormal basis functions $\vartheta_{j}(\mathbf{r})$, with $j=1, \ldots, \hat{n}$, is then determined as

$$
\vartheta_{j}(\mathbf{r})=\sum_{i=1}^{\hat{n}} v_{i}(\mathbf{r}) u_{i j} / \sqrt{d_{j}} .
$$

Each basis functions $\vartheta_{j}(\mathbf{r})$, with $j=1, \ldots, \hat{n}$ is associated to a singular value $\sqrt{d_{j}}$. As a consequence of the characteristics of the SVD decomposition, for a sufficiently small value of the parameter $\varepsilon$, the basis functions $\vartheta_{j}(\mathbf{r})$ associated to the singular values $\sqrt{d_{j}}$ such that $\sqrt{d_{j} / d_{1}}<\varepsilon$, can be disregarded, without sacrificing the accuracy in the approximation (18). As verified by numerical experiments in Section $\mathrm{V}$, values of $\varepsilon$ larger that $10^{-6}$ can be assumed. In this way, the number of basis functions is further reduced from $\hat{n}$ to $\hat{n}_{\vartheta}$. Analogous considerations can be repeated for constructing the basis functions $\lambda_{j}(\mathbf{r})$, with $j=1, \ldots, \hat{n}_{\lambda}$. As verified by numerical experiments in Section $\mathrm{V}$, in order to get accurate approximations of the space-time evolution of temperature, $\hat{n}_{\vartheta}$ and $\hat{n}_{\lambda}$ not larger than 25 can be assumed. As a result, DCTMs of small statespace dimensions are achieved. The simulation of such DCTMs hence implies negligible computational cost with respect to a conventional FEM approach.

\section{CAse Studies in UTCS Technology}

Emerging multichip technologies provide an opportunity to increase the integration density of semiconductor systems so as to yield smaller, lighter, and cheaper products. Nowadays, extremely dense modules are fabricated in UTCS technology, which exploits the recent advances in wafer thinning, as well as in attachment, bonding, and interconnection. In UTCS systems, multiple silicon chips thinned down to $10 \mu \mathrm{m}$ are vertically integrated on a single (inactive) host silicon substrate, being the electrical insulation among them ensured by 


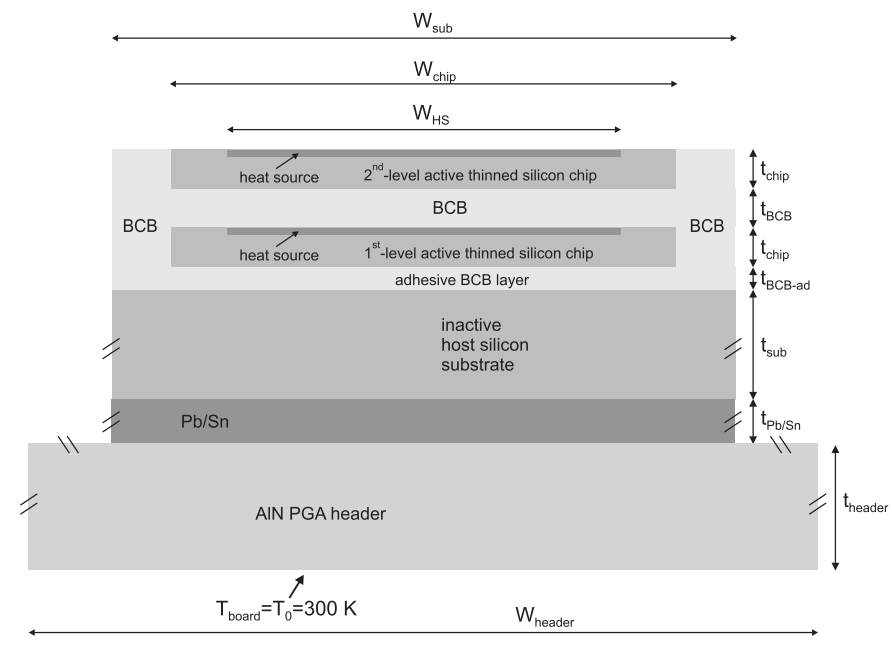

Fig. 1. Cross-section of the stacked two-chip UTCS module under test (not to scale).

layers of benzocyclobutene (BCB), a photosensitive polymer with good planarization properties [11], [12], [29], [30]; the resulting stack provides larger circuitry integration than in 2-D ICs. Unfortunately, UTCS architectures may be subject to exacerbated thermal effects dictated by (i) the high power density and (ii) the low thermal conductivity of BCB (about 800 times lower than that of silicon), which inhibits the downward heat propagation from the power-dissipating regions to the board [11], [12], [30]. As a consequence, the potential benefits of stacked architectures can in principle be achieved only by resorting to thermal-aware design techniques based on reliable simulations. An extensive numerical analysis of the thermal behavior of typical UTCS modules has been recently carried out [13], [31]; among other results, the detrimental impact of the $\mathrm{BCB}$ on the vertical heat flow was quantified.

The procedure addressed in previous sections for determining one-port DCTMs is here applied to an UTCS structure containing two $10-\mu \mathrm{m}$-thick silicon chips, as shown in Fig. 1. The upper $\left(2^{\text {nd }}-\right.$ level $)$ chip is vertically insulated from the buried $\left(1^{\text {st }}-\right.$ level $)$ one by a BCB planarization layer; the lower chip is attached to the host silicon substrate by an adhesive BCB layer. The power-dissipating circuitries (i.e., the heat sources) lie on the top of the chips. The substrate backside is soldered to the AlN package header by a $\mathrm{Pb} / \mathrm{Sn}$ conductive grease. The through-silicon vias (TSVs) needed to ensure the vertical interconnectivity between chips [29] were not accounted for in the analysis due to their small size that only slightly favor the downward heat flow [11]. In addition, heat spreading solutions like the adoption of nonsignal TSVs [30], as well as of a copper grid or plate [11], have not been considered with the aim of modeling a test chip significantly suffering from thermal effects. The header is assumed to be in close contact with the board at ambient temperature $\left(T_{\text {board }}=T_{0}\right)$. The horizontal and vertical dimensions of the module are reported in Table II. The analysis was performed by assuming adiabatic top and lateral faces of the domain, which is reasonable due to the small influence of natural convection [13]. It is noted that only one quarter of the structure was simulated, the missing portion being virtually
TABLE II

VALUeS OF THE GeOMETRICAL PARAMETERS INDICATED IN FIG. 1

\begin{tabular}{|c|c|}
\hline Parameter & Value $[\mu \mathrm{m}]$ \\
\hline$W_{\text {header }}$ & 15000 \\
$W_{\text {sub }}$ & 6200 \\
$W_{\text {chip }}$ & 5620 \\
$W_{\mathrm{HS}}$ & 4240 \\
$t_{\text {chip }}$ & 10 \\
$t_{\mathrm{BCB}}$ & 10 \\
$t_{\mathrm{BCB}-\mathrm{ad}}$ & 3 \\
$t_{\text {sub }}$ & 500 \\
$t_{\mathrm{Pb} / \mathrm{Sn}}$ & 50 \\
$t_{\text {header }}$ & 760 \\
\hline
\end{tabular}

TABLE III

VAlues of the Material Parameters

\begin{tabular}{|l|c|c|c|c|c|}
\hline Material & $\begin{array}{c}\rho \\
{\left[\mathrm{kg} / \mu \mathrm{m}^{3}\right]}\end{array}$ & $\begin{array}{c}c \\
{[\mathrm{~J} / \mathrm{kgK}]}\end{array}$ & $\begin{array}{c}k_{0} \\
{[\mathrm{~W} / \mu \mathrm{mK}]}\end{array}$ & $\begin{array}{c}k_{1} \\
{[\mathrm{~W} / \mu \mathrm{mK}]}\end{array}$ & $m$ \\
\hline $\mathrm{Si}[33]-[36]$ & $2.330 \cdot 10^{-15}$ & 711 & $1.48 \cdot 10^{-4}$ & $1.48 \cdot 10^{-4}$ & -1.33 \\
\hline A) BCB [11] & $1.051 \cdot 10^{-15}$ & 1267 & $1.80 \cdot 10^{-7}$ & 0 & 0 \\
B) BCB [32] & $1.051 \cdot 10^{-15}$ & 1267 & $1.80 \cdot 10^{-7}$ & $3.00 \cdot 10^{-7}$ & 1 \\
\hline $\mathrm{Pb} / \mathrm{Sn}[37]$ & $11.200 \cdot 10^{-15}$ & 137 & $0.36 \cdot 10^{-4}$ & $0.36 \cdot 10^{-4}$ & -1.10 \\
\hline $\mathrm{AlN}[38]$ & $3.260 \cdot 10^{-15}$ & 748 & $1.50 \cdot 10^{-4}$ & $1.50 \cdot 10^{-4}$ & -1.57 \\
\hline
\end{tabular}

recreated by applying adiabatic boundary conditions over the planes of symmetry.

Table III gives the material parameters adopted for the analysis. Two cases were analyzed: 1) case A, in which the thermal conductivity of $\mathrm{BCB}$ was considered to be temperature-insensitive, as assumed in [11], and 2) case B, in which it was modeled as linearly increasing with temperature on the basis of the experimental data shown in [32]. Both cases are nonlinear since the temperature dependences of the thermal conductivities of $\mathrm{Si}, \mathrm{Pb} / \mathrm{Sn}$, and $\mathrm{AlN}$ are accounted for. The dissipated power density was assumed uniform within each heat source.

A 3-D tetrahedral mesh of the structure was built by the software package COMSOL [39]. Such a mesh, shown in Fig. 2, was rather cumbersome to generate due to the presence of layers with thickness much lower than horizontal dimensions and than thicknesses of other layers; it was created with smart selective refinement strategies-available in the recent software releases-and includes more than 1 million elements (tetrahedra). This COMSOL mesh is used for the 


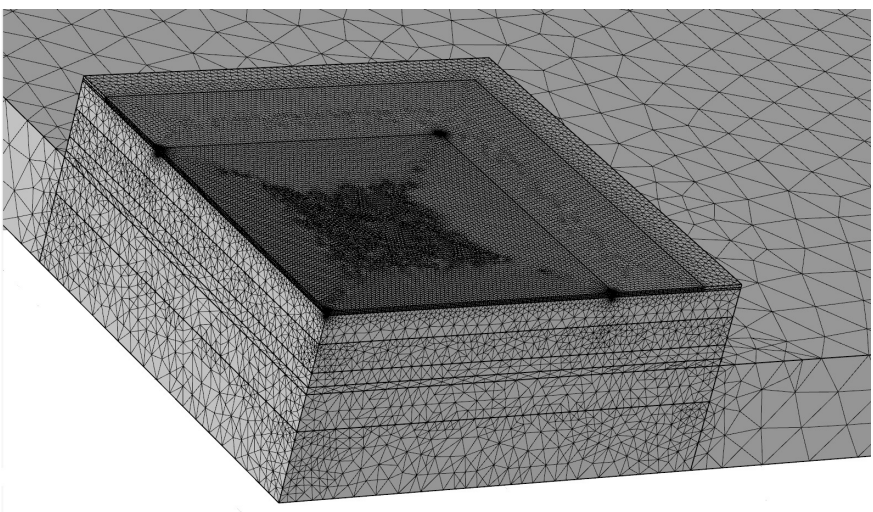

Fig. 2. Detail of the COMSOL mesh corresponding to the analyzed UTCS structure.

second-order FEM discretization of the linear heat diffusion problems (36) and (37), by means of about 1.5 million DoFs.

First, the case in which only the $2^{\text {nd }}$-level circuitry is active was considered. The expansion points $\sigma_{m}$ and multiindexes $\boldsymbol{\alpha}_{m}$, with $m=1, \ldots, r$, were chosen as described in Section IV, with $l=10$ and $r=3$. As a result, $l r=30$ discretized linear heat diffusion problems in the frequency domain were solved by the conjugate gradient algorithm with incomplete Choleski preconditioning. This took less than $1 \mathrm{~h} 15 \mathrm{~min}$ and less than $1 \mathrm{~GB}$ of RAM storage on a 2.3 Ghz Intel Core i7. By applying the SVD algorithm, with $\varepsilon=10^{-5}$, a DCTM with $\hat{n}_{\vartheta}=\hat{n}_{\lambda}=12$ was obtained.

The resulting model can be exploited for determining the thermal behavior for any waveform of power $P(t)$. Here, the case of a constantly dissipated power density $q=5 \mu \mathrm{W} / \mu \mathrm{m}^{3}$ (i.e., $P \approx 45 \mathrm{~W}$ ) is analyzed by comparing the results provided by the DCTM with those calculated by COMSOL. The numerical solution of the DCTM by means of the ode15s MATLAB solver requires less than $2 \mathrm{~s}$, to be compared with about $11 \mathrm{~h}$ needed by COMSOL. This means that the computational time for constructing and solving a DCTM is about one order of magnitude lower than that elapsed for a single 3-D FEM transient simulation. It is noted that the 3-D FEM simulation had to be carried out with low tolerances in order to guarantee the same accuracy provided by the DCTM, and required about 10 GB of memory storage. Fig. 3(a) shows the resulting thermal impedances $Z_{\mathrm{TH}}(t)$ for the fully linear case (i.e., that obtained by disregarding all the temperature dependences of the thermal conductivities), for the modified linear solution through the Kirchhoff and Batty et al.'s transformations, and for the nonlinear cases A and B. Fig. 3(b) depicts the profile of the temperature rise along the vertical axis crossing the center of the module. These figures illustrate that the DCTM is suited to describe not only the thermal impedance $Z_{\mathrm{TH}}(t)$, but also the whole space-time evolution of the temperature rise with great accuracy, the discrepancy with respect to 3 -D FEM data being lower than $0.2 \%$. From these results, various observations are in order.

1) The thermal resistances $R_{\mathrm{TH}}$ are equal to $3.77,3.88$, and $2.90 \mathrm{~K} / \mathrm{W}$ for the linear and the nonlinear cases $\mathrm{A}$ and $\mathrm{B}$, respectively. In spite of the low $\mathrm{BCB}$
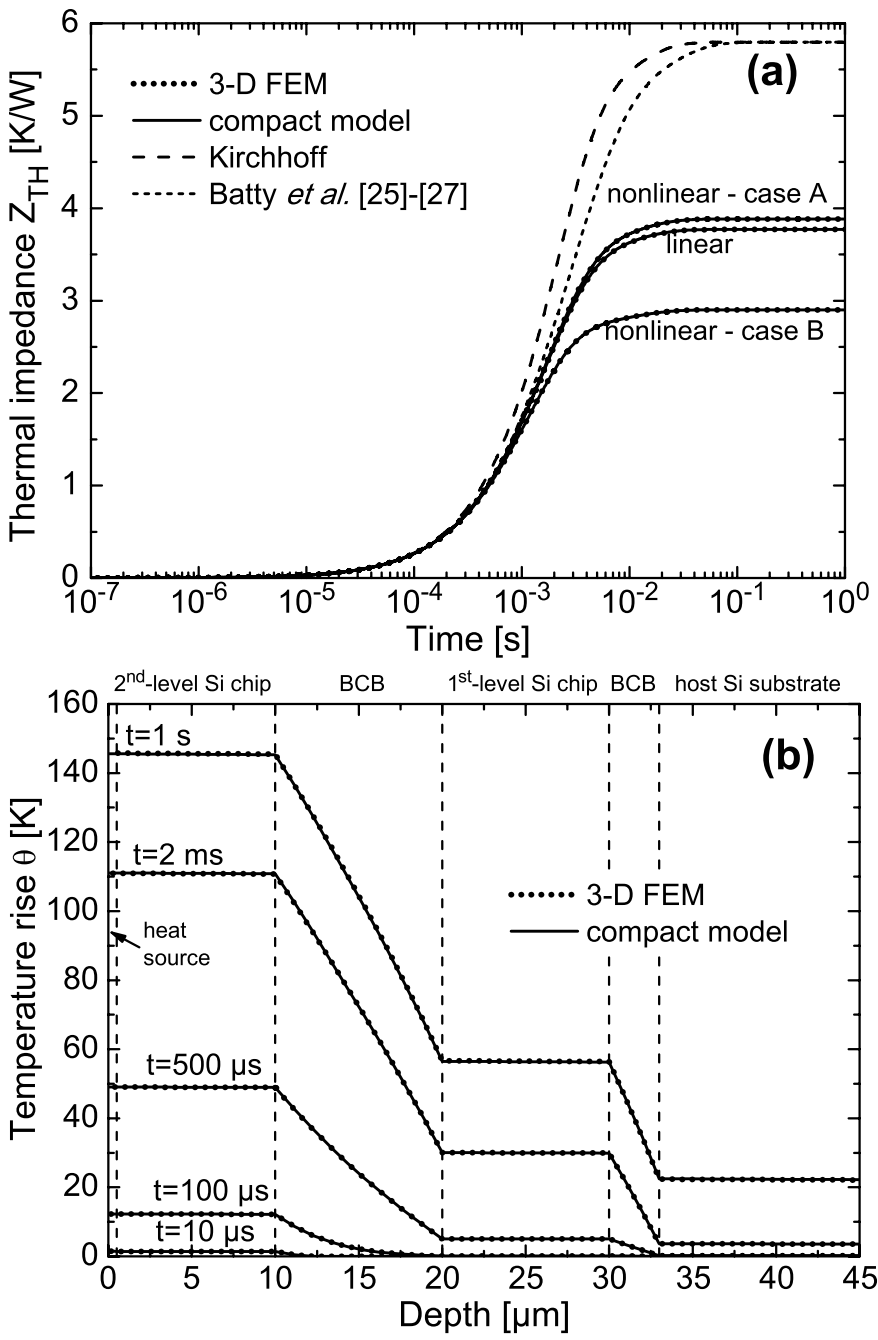

Fig. 3. Case of activation of the circuitry lying on the upper silicon chip. (a) Thermal impedance against time for the linear and nonlinear cases, as simulated by COMSOL (dotted lines) and obtained through the compact model (solid), along with the evolution determined by the mere application of the Kirchhoff's (dashed) and Batty et al.'s (short-dashed) transformations with the temperature dependence of Si. (b) Vertical profile of the temperature rise along the module for the nonlinear case $\mathrm{B}$, as computed by COMSOL (dotted lines) and the compact model (solid) at five time instants.

conductivity, the values are not high due to the large heat source area.

2) It can be easily inferred that the $\mathrm{BCB}$ dominates the thermal behavior. In case $\mathrm{B}, k_{\mathrm{BCB}}$ linearly grows from $1.8 \times 10^{-7} \mathrm{~W} / \mu \mathrm{mK}$ at $T=300 \mathrm{~K}$ to $3.25 \times 10^{-7} \mathrm{~W} / \mu \mathrm{mK}$ at $T=425 \mathrm{~K}$, thus leading to a cooling action high enough to prevail over the heating mechanism induced by the reduction in thermal conductivity of other materials; as a result, the thermal behavior of the module improves compared with the linear case. The large discrepancies between the results corresponding to the two cases A and B suggest that care must be taken in modeling the temperature dependence of the thermal conductivities of all materials belonging to the structure under test.

3) An inspection of the figure reveals the dramatic inaccuracy obtained by merely applying the Kirchhoff's 

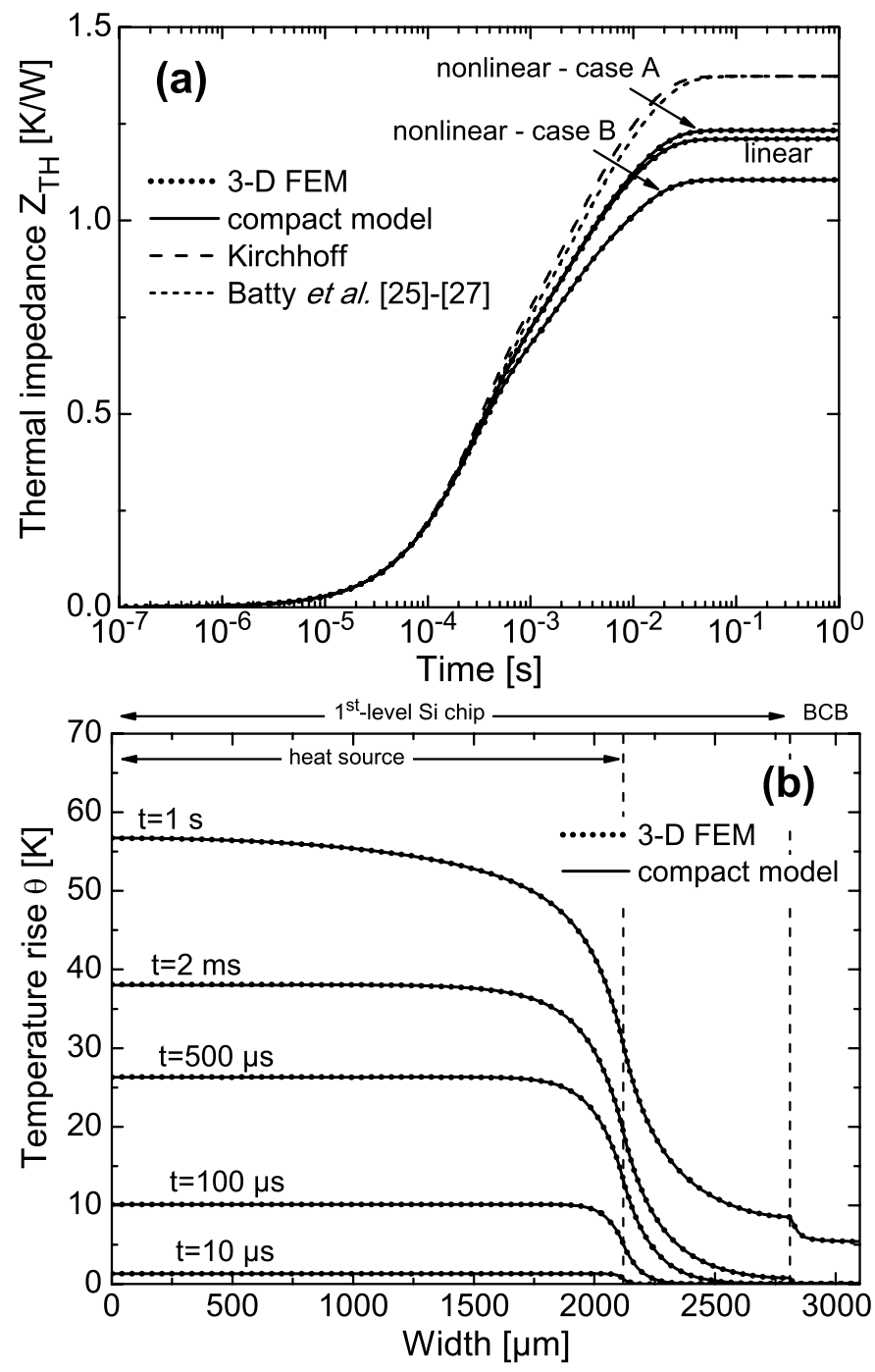

Fig. 4. Case of activation of the circuitry located on the buried silicon chip. (a) Thermal impedance versus time for the linear and nonlinear cases, as simulated by COMSOL (dotted lines) and determined through the compact model (solid), along with the evolution obtained by applying the Kirchhoff's (dashed) and Batty et al.'s (short-dashed) transformations based on the temperature dependence of the thermal conductivity of Si. (b) Horizontal profile of the temperature rise along the module for the nonlinear case B, as evaluated by COMSOL (dotted lines) and the compact model (solid) at five time instants.

transformation based on the law relating the thermal conductivity of $\mathrm{Si}$ to temperature, i.e., by using (12) with $m=-1.33$; in particular, $R_{\mathrm{TH}}$ differs by $\sim 50 \%$ and $\sim 100 \%$ from the exact values associated to the nonlinear cases A and B. Including the further time variable transformation by Batty et al. only a marginal improvement is gained for medium times, while the steady-state results remain unchanged.

A similar analysis was repeated by activating only the circuitry (the heat source) embedded in the $1^{\text {st }}$-level chip. Fig. 4(a) shows that in this case the self-heating is considerably mitigated since the buried chip is closer to the board kept at ambient temperature. In particular, it is found that the thermal resistances $R_{\mathrm{TH}}$ amount to $1.21,1.23$, and $1.10 \mathrm{~K} / \mathrm{W}$ for the linear and nonlinear cases A and B, respectively. It is worth noting that the plain reduction in the curve slope

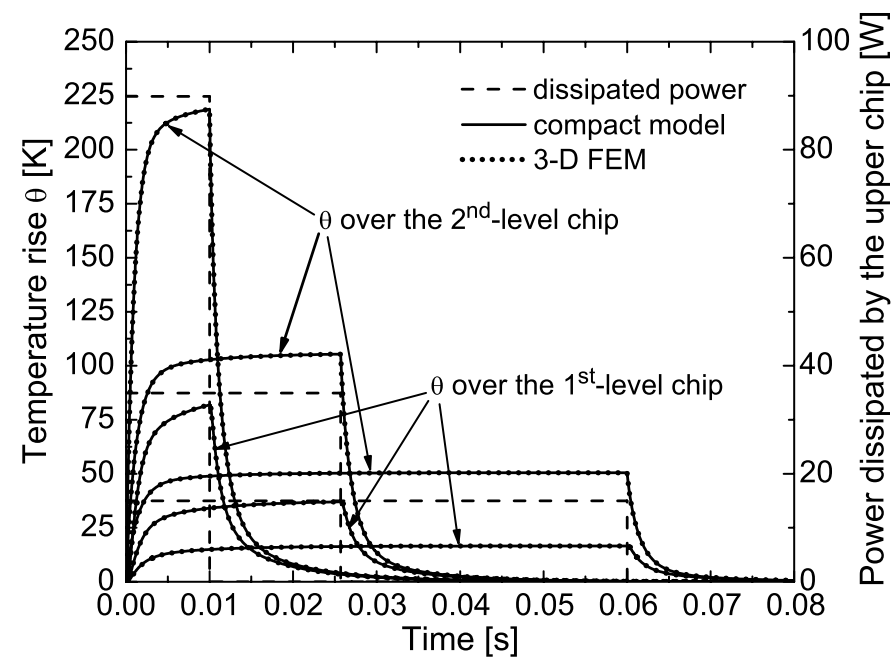

Fig. 5. (Left) Average temperature rises over the upper and buried silicon chips induced by (right) three profiles of power dissipated by the circuitry located on the $2^{\text {nd }}$-level chip (dashed lines), as determined through COMSOL (dotted) and the compact model (solid) for the nonlinear case B.

triggered by the heat reaching the silicon substrate takes place earlier than in the case with the $2^{\text {nd }}$-level circuitry activated. All the findings reported above concerning the accuracy of the compact model and the required CPU times and RAM storage still hold true in this case. This is evidenced in Fig. 4(b), which depicts the profile of the temperature rise along a horizontal axis crossing the center of the module, as computed by both COMSOL and the DCTM.

Fig. 5 witnesses that the DCTM approach allows accurately following the temperature evolutions induced by short activations of the circuitry located on the chips-which are inherently cumbersome to be simulated at the edges of the square-wave power profiles (i.e., when the heat source turns ON and OFF) if exploiting conventional 3-D FEM tools. In particular, three power pulses dissipated by the upper chip are considered, which are characterized by different levels and durations so as to keep constant the pulse area. The figure shows the transient behavior of the temperature rise averaged over both the circuitries, as evaluated by the DCTM and COMSOL for the nonlinear case B. Consistently with the previous analyses, it is found that the approaches provide almost identical results, although for each power profile the DCTM simulation lasts less than $2 \mathrm{~s}$ while COMSOL requires more than $20 \mathrm{~h}$ mainly due to the aforementioned issue.

\section{CONCLUSION}

In this paper, a novel approach has been proposed for constructing DCTMs of nonlinear heat diffusion problems. The method leads to compact models of small state-space dimensions. The whole space-time evolution of the temperature field can be accurately determined regardless of the geometrical complexity of the domain, as well as of the power waveform. The method has been adopted to gain precious information on the thermal behavior of state-of-the-art UTCS modules, which are composed by individual layers with highly different (i) thermal conductivities and (ii) thermal conductivity dependences on temperature. It has been found that the 
computational time to construct each DCTM is one order of magnitude lower than the one elapsed for a conventional transient 3-D FEM simulation. It can be concluded that this approach can be successfully used as a viable alternative to commercial numerical tools to support the thermal design of electronic structures where nonlinear thermal effects play an important role.

\section{REFERENCES}

[1] M.-N. Sabry, "Dynamic compact thermal models used for electronic design: A review of recent progress," in Proc. IPACK, Maui, HI, USA, 2003, pp. 399-415.

[2] T. Azoui, P. Tounsi, and J.-M. Dorkel, "Innovative methodology to extract dynamic compact thermal models: Application to power devices," in Proc. IEEE 16th Int. Workshop Thermal Investigat. ICs Syst. (THERMINIC), Oct. 2010, pp. 1-5.

[3] E. Monier-Vinard, C. T. Dia, V. Bissuel, N. Laraqi, and O. Daniel, "Dynamic compact thermal model for stacked-die components," in Proc. 28th Annu. IEEE Semicond. Thermal Meas. Manage. Symp. (SEMI-THERM), Mar. 2012, pp. 20-28.

[4] M.-N. Sabry and M. Dessouky, "A framework theory for dynamic compact thermal models," in Proc. 28th Annu. IEEE Semicond. Thermal Meas. Manage. Symp. (SEMI-THERM), Mar. 2012, pp. 189-194.

[5] L. Codecasa, D. D'Amore, and P. Maffezzoni, "An Arnoldi based thermal network reduction method for electro-thermal analysis," IEEE Trans. Compon. Packag. Technol., vol. 26, no. 1, pp. 186-192, Mar. 2003.

[6] L. Codecasa, D. D'Amore, and P. Maffezzoni, "Compact modeling of electrical devices for electrothermal analysis," IEEE Trans. Circuits Syst. I, Fundam. Theory Appl., vol. 50, no. 4, pp. 465-476, Apr. 2003.

[7] L. Codecasa, D. D'Amore, and P. Maffezzoni, "Compact thermal networks for modeling packages," IEEE Trans. Compon. Packag. Technol., vol. 27, no. 1, pp. 96-103, Mar. 2004.

[8] L. Codecasa, D. D'Amore, P. Maffezzoni, and W. Batty, "Analytical multipoint moment matching reduction of distributed thermal networks," IEEE Trans. Compon. Packag. Technol., vol. 27, no. 1, pp. 87-95, Mar. 2004.

[9] L. Codecasa, "Compact models of dynamic thermal networks with many heat sources," IEEE Trans. Compon. Packag. Technol., vol. 30, no. 4, pp. 653-659, Dec. 2007.

[10] M. Rencz and V. Szèkely, "Studies on the nonlinearity effects in dynamic compact model generation of packages," IEEE Trans. Compon. Packag. Technol., vol. 27, no. 1, pp. 124-130, Mar. 2004.

[11] S. Pinel et al., "Thermal modeling and management in ultrathin chip stack technology," IEEE Trans. Compon. Packag. Technol., vol. 25, no. 2, pp. 244-253, Jun. 2002.

[12] J. Palacín, M. Salleras, J. Samitier, and S. Marco, "Dynamic compact thermal models with multiple power sources: Application to an ultrathin chip stacking technology," IEEE Trans. Adv. Packag., vol. 28, no. 4, pp. 694-703, Nov. 2005.

[13] N. Rinaldi, S. Russo, and V. d'Alessandro, "Thermal effects in thin silicon dies: Simulation and modelling," in Ultra-Thin Chip Technology and Applications, J. Burghartz, Ed. New York, NY, USA: Springer-Verlag, 2010, ch. 23.

[14] H. S. Carlslaw and J. C. Jaeger, Conduction of Heat in Solids, 2nd ed. New York, NY, USA: Oxford Univ. Press, 1959, sec. I.1.6.

[15] M. Necati Özisik, Boundary Value Problems of Heat Conduction. New York, NY, USA: Dover, 1989, sec. 8.2.

[16] J. R. Phillips, "Projection-based approaches for model reduction of weakly nonlinear, time-varying systems," IEEE Trans. Comput.-Aided Design Integr. Circuits Syst., vol. 22, no. 2, pp. 171-187, Feb. 2003.

[17] M. Rewieński and J. White, "A trajectory piecewise-linear approach to model order reduction and fast simulation of nonlinear circuits and micromachined devices," IEEE Trans. Comput.-Aided Design Integr. Circuits Syst., vol. 22, no. 2, pp. 155-170, Feb. 2003.

[18] P. Li and L. T. Pileggi, "Compact reduced-order modeling of weakly nonlinear analog and RF circuits," IEEE Trans. Comput.-Aided Design Integr. Circuits Syst., vol. 24, no. 2, pp. 184-203, Feb. 2005.

[19] C. Gu, "QLMOR: A projection-based nonlinear model order reduction approach using quadratic-linear representation of nonlinear systems,' IEEE Trans. Comput.-Aided Design Integr. Circuits Syst., vol. 30, no. 9, pp. 1307-1320, Sep. 2011.

[20] L. Codecasa, "Novel approach to compact modeling for nonlinear thermal conduction problems," in Proc. IEEE 19th Int. Workshop Thermal Investigat. ICs Syst. (THERMINIC), Sep. 2013, pp. 164-169.
[21] L. Codecasa, "Nonlinear dynamic compact thermal models by structure-preserving projection," Microelectron. J., 2014, doi: 10.1016/j.mejo.2014.06.001.

[22] W. J. Rugh, Nonlinear System Theory: The Volterra/Wiener Approach. Baltimore, MD, USA: Johns Hopkins Univ. Press, 1981.

[23] L. Codecasa, "Canonical forms of one-port passive distributed thermal networks," IEEE Trans. Compon. Packag. Technol., vol. 28, no. 1, pp. 5-13, Mar. 2005

[24] W. B. Joyce, "Thermal resistance of heat sinks with temperaturedependent conductivity," Solid-State Electron., vol. 18, no. 4, pp. 321-322, 1975.

[25] W. Batty and C. M. Snowden, "Electro-thermal device and circuit simulation with thermal nonlinearity due to temperature dependent diffusivity," Electron. Lett., vol. 36, no. 23, pp. 1966-1968, Nov. 2000.

[26] K. Krabbenhoft and L. Damkilde, "Comments on 'Electro-thermal device and circuit simulation with thermal nonlinearity due to temperature dependent diffusivity'," Electron. Lett., vol. 37, no. 24, pp. 1481-1482, Nov. 2001.

[27] W. Batty, S. David, and C. M. Snowden, "Reply to Comment on 'Electro-thermal device and circuit simulation with thermal nonlinearity due to temperature dependent diffusivity'," Electron. Lett., vol. 37, no. 24, pp. 1482-1483, Nov. 2001.

[28] T. Hélie and B. Laroche, "On the convergence of Volterra series of finite dimensional quadratic MIMO systems," Int. J. Control, vol. 81, no. 3 pp. 356-368, 2008.

[29] F. Iker et al., "3D embedding and interconnection of ultra thin $(\ll 20 \mu \mathrm{m})$ silicon dies," in Proc. IEEE 9th Electron. Packag. Technol. Conf., Dec. 2007, pp. 222-226.

[30] A. Ziabari and A. Shakouri, "Fast thermal simulations of vertically integrated circuits (3D ICs) including thermal vias," in Proc. 13th IEEE Intersoc. Conf. Thermal Thermomech. Phenomena Electron. Syst. (ITherm), May/Jun. 2012, pp. 588-596.

[31] V. d'Alessandro, M. de Magistris, A. Magnani, N. Rinaldi, and S. Russo, "Dynamic electrothermal macromodeling: An application to signal integrity analysis in highly integrated electronic systems," IEEE Trans. Compon., Packag., Manuf. Technol., vol. 3, no. 7, pp. 1237-1243, Jul. 2013.

[32] X. Xu and Z. Wang, "Thermal conductivity enhancement of benzocyclobutene with carbon nanotubes for adhesive bonding in 3-D integration," IEEE Trans. Compon., Packag., Manuf. Technol., vol. 2, no. 2, pp. 286-293, Feb. 2012.

[33] F. Bonani and G. Ghione, "On the application of the Kirchhoff transformation to the steady-state thermal analysis of semiconductor devices with temperature-dependent and piecewise inhomogeneous thermal conductivity," Solid-State Electron., vol. 38, no. 7, pp. 1409-1412, 1995.

[34] K. J. Negus, R. W. Franklin, and M. M. Yovanovich, "Thermal modeling and experimental techniques for microwave bipolar devices," IEEE Trans. Compon., Hybrids, Manuf. Technol., vol. 12, no. 4, pp. 680-689, Dec. 1989.

[35] N. Rinaldi, "Small-signal operation of semiconductor devices including self-heating, with application to thermal characterization and instability analysis," IEEE Trans. Electron Devices, vol. 48, no. 2, pp. 323-331, Feb. 2001.

[36] J. C. J. Paasschens, S. Harmsma, and R. van der Toorn, "Dependence of thermal resistance on ambient and actual temperature," in Proc. IEEE Bipolar/BiCMOS Circuits Technol. Meeting (BCTM), Sep. 2004, pp. 96-99.

[37] Y.-F. Chiu, Y.-L. Tsai, and W.-S. Hwang, "Mathematical modeling for the solidification heat-transfer phenomena during the reflow process of lead-tin alloy solder joint in electronics packaging," Appl. Math. Modelling, vol. 27, no. 7, pp. 565-579, 2003.

[38] V. Palankovski, Analysis and Simulation of Heterostructure Devices. New York, NY, USA: Springer-Verlag, 2004.

[39] Comsol Multiphysics 3.5a, User's Manual, COMSOL AB, Stockholm, Sweden, 2008. 